\title{
Contribución al estudio microscópico de la estructura del clínker de cemento portland. I
}

\author{
J. CALleJA* Y J. M. FERnANDEZ PARIS**
}

\section{regsumem}

Existen en el clínker de cemento portland heterogeneidades en cuanto a la composición química de sus distintas zonas. Estas heterogeneidades hacen sospechar diferencias de constitución, que aparecen como resultado del cálculo potencial por zonas. Los métodos químicos son insuficientes para poner de manifiesto por vía experimental esas diferencias. Es preciso recurrir para ello a técnicas de naturaleza física.

En el presente trabajo se utiliza la técnica microscópica petrográfica y se desarrolla un método experimental de observación y recuento que permite lograr de forma original el fin propuesto.

Las diferencias de constitución mineralógica del clínker en sus distintas zonas pueden ser relacionadas con particularidades inherentes a las materias primas y al proceso de fabricación. Esto permite efectuar, por vía microscópica, una diagnosis de las vicisitudes de dicho proceso, intimamente ligadas a su vez a las características tecnológicas del cemento obtenido de cada clínker estudiado. El conocimiento asi adquirido permite influir a voluntad y conveniencia en el proceso de fabricación del clínker.

\section{ANTECEDENTES}

El clínker de cemento portland es heterogéneo en cuanto a su composición química y a su constitución mineralógica o composición potencial. Es decir, expresada la composición química en tantos por ciento de óxidos y la potencial calculada a partir de la química, en tantos por ciento de especies mineralógicas (silicatos, aluminatos y ferritos) correspondientes al sistema cuaternario $\mathrm{CaO}-\mathrm{SiO}_{2}-\mathrm{Al}_{2} \mathrm{O}_{3}-\mathrm{Fe}_{2} \mathrm{O}_{3}$ (abreviadamente C-S-A-F), se observan heterogeneidades en la distribución de los diferentes conceptos analíticos según distintas zonas (periférica o cortical, intermedia, e interna o nuclear) consideradas en los gránulos o nódulos de clínker.

* Dr. en Ciencias Quimicas. Investigador Cientifico y Jefe del Departamento de Química del I.E.T.c.c. ** Ingeniero Técnico Químico, del citado Departamento del I.E.T.c.c. 
Estas heterogeneidades, por lo que respecta a la composición expresada en óxidos, han sido puestas claramente de manifiesto por vía química en una serie de trabajos experimentales previos. Así, por ejemplo, ha quedado establecido el hecho general de la mayor abundancia de cal libre en la parte interna de los gránulos (1)*. En cuanto a los álcalis (óxidos sódico y potásico) también tienen tendencia a acumularse en el interior de los gránulos, particularmente por lo que se refiere al óxido potásico que es el más abundante (2).

La heterogénea distribución de la cal libre y de los álcalis, y particularmente la de la primera, hacen prever heterogeneidades en la distribución de los óxidos de carácter ácido $\left(\mathrm{SiO}_{2}, \mathrm{Al}_{2} \mathrm{O}_{3}\right.$ y $\left.\mathrm{Fe}_{2} \mathrm{O}_{3}\right)$ y, dentro de éstos, en la de los fundentes $\left(\mathrm{Al}_{2} \mathrm{O}_{3}\right.$ y $\mathrm{Fe}_{2} \mathrm{O}_{3}$; en conjunto $\mathrm{R}_{2} \mathrm{O}_{3}$ ). En efecto, tales heterogeneidades han sido puestas de relieve (3) en el sentido de que en las zonas corticales de los gránulos de clínker, junto a un menor contenido de óxido cálcico libre y de álcalis, respecto de las zonas internas, existe también una mayor proporción de óxidos alumínico y férrico $\left(\mathrm{R}_{2} \mathrm{O}_{3}=\mathrm{Al}_{2} \mathrm{O}_{3}+\mathrm{Fe}_{2} \mathrm{O}_{3}\right)$. También el contenido de sílice (anhídrido silícico $\mathrm{SiO}_{2}$ ) crece hacia el interior de los gránulos. El óxido cálcico total (combinado y libre) apenas presenta variaciones muy ligeras que, además, son signo vario.

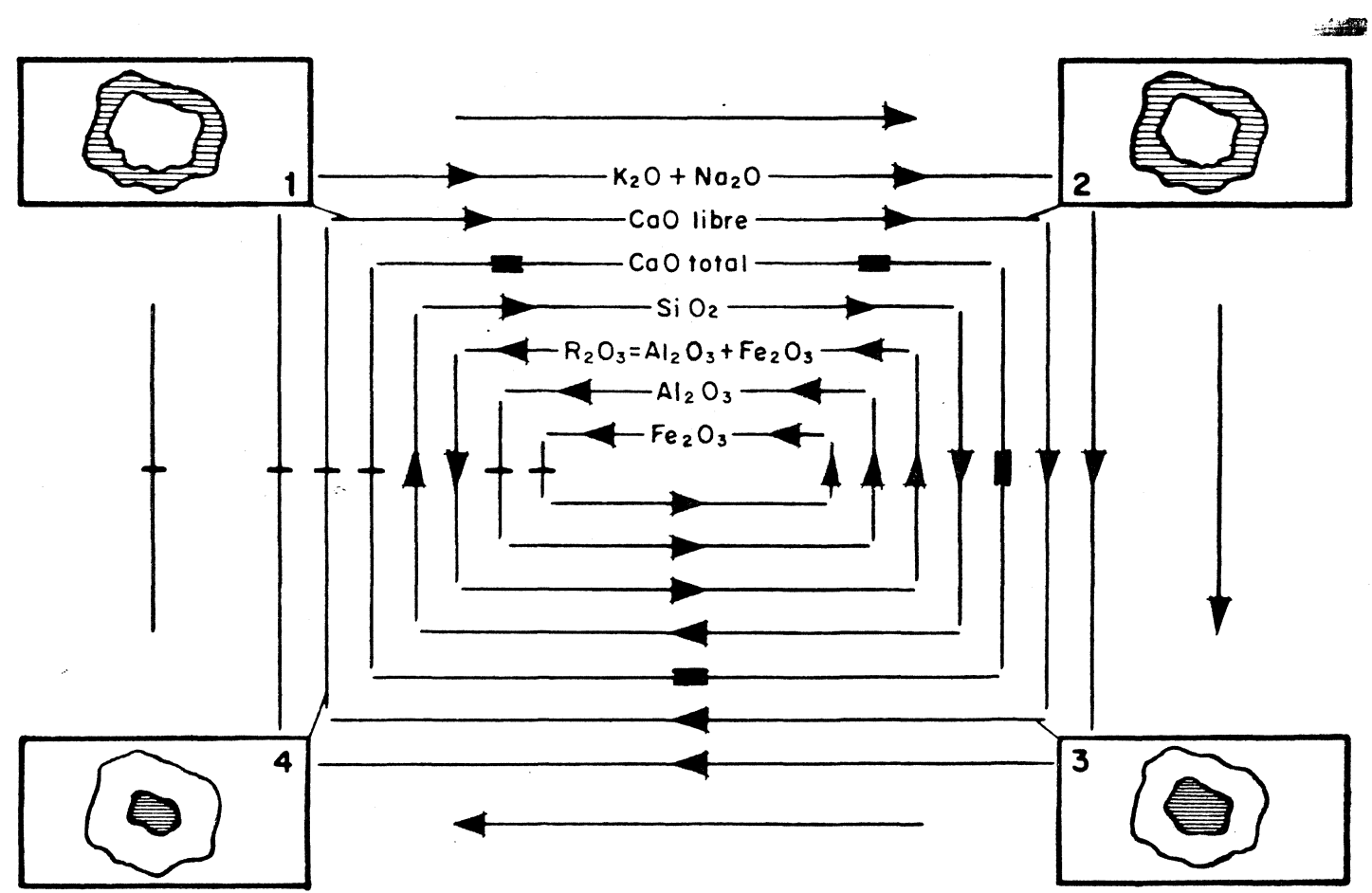

Fig. 1

Los resultados de cuanto antecede pueden representarse de forma gráfica en el esquema de la figura 1.

Estas heterogeneidades en cuanto-a la composición química expresada en óxidos permiten predecir otras relativas a la composición potencial calculada a partir de aquélla, y que el propio cálculo potencial pone de manifiesto. Tales heterogeneidades en la distribución zonal

* Las llamadas como la precedente corresponden a la bibliografía inserta al final del articulo. 
de los componentes potenciales consisten globalmente en lo siguiente (2) y (3): un mayor contenido de silicato tricálcico $\mathrm{C}_{3} \mathrm{~S}$, de aluminato tricálcico $\mathrm{C}_{3} \mathrm{~A}$, de ferritoaluminato tetracálcico $\mathrm{C}_{4} \mathrm{AF}$ y de fase líquida (o vítrea) en las zonas periféricas, acompañado de una menor abundancia de silicato bicálcico $\mathrm{C}_{2} \mathrm{~S}$ y de silicato bicálcico con óxido potásico en disolución sólida $\mathrm{KC}_{23} \mathrm{~S}_{12}$. Naturalmente que en las zonas internas se invierten los términos.

Estos resultados pueden observarse de forma gráfica en el esquema de la figura 2 .

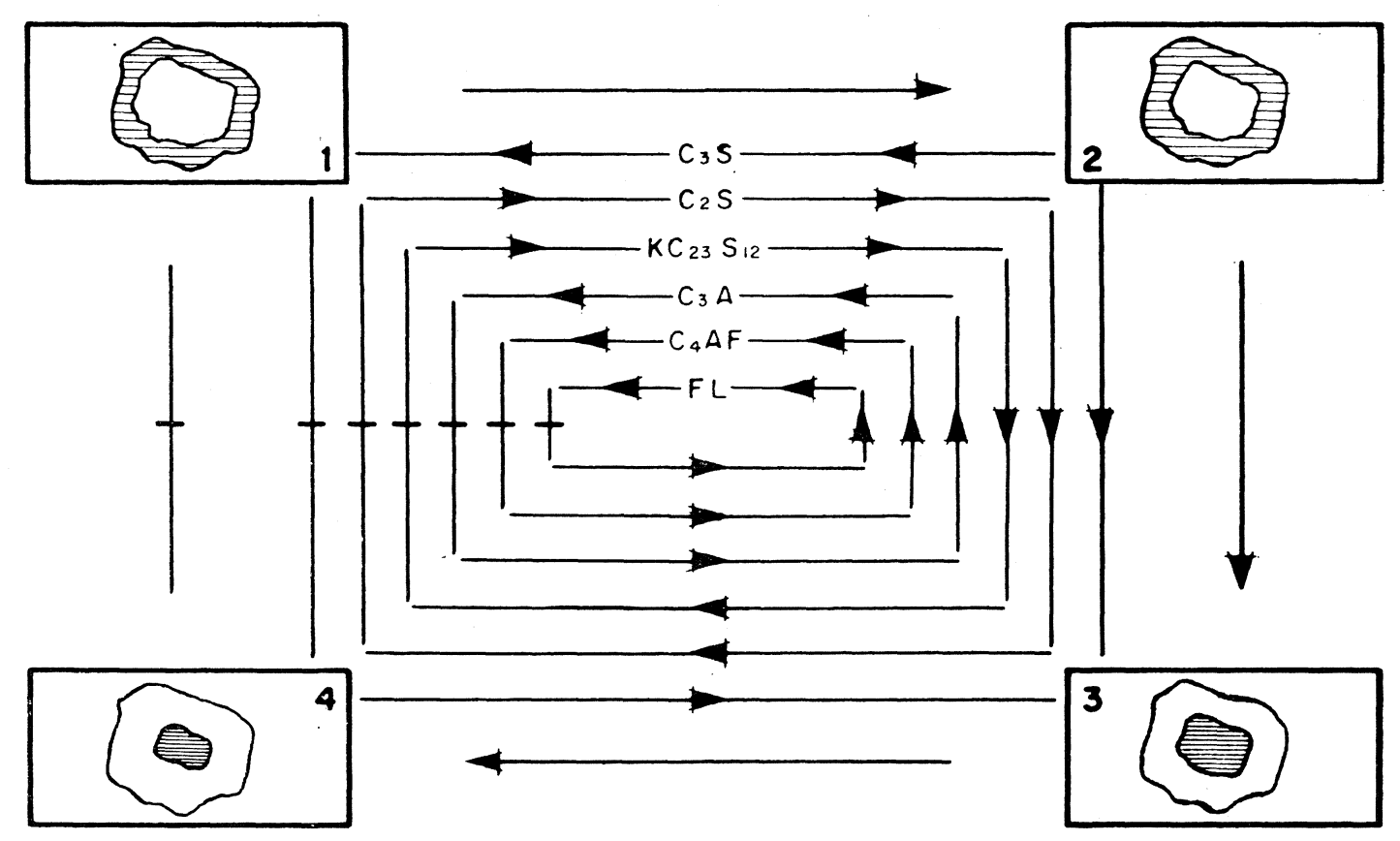

Fig. ?

Consecuentemente con estos resultados pueden establecerse unas relaciones análogas por lo que se refiere a los índices y módulos químicos del clínker. Así, por ejemplo, el módulo hidráulico de Michaelis $\mathrm{MH}$ apenas varía de las zonas periféricas a las interiores, mientras que el módulo silícico de Kühl MS es bastante menor en las primeras que en las segundas, y el módulo alumínico, férrico o de fundentes MF y el grado de saturación de cal de Kühl GS, son algo mayores.

Estas relaciones pueden observarse gráficamente en el esquema de la figura 3.

Todos los resultados anteriores y algunos otros logrados por una modificación de la técnica operatoria en relación con (1) y (3) pueden resumirse de manera gráfica en el esquema de la figura 4 (2) y (4).

Sobre la base de los resultados puestos de manifiesto por los esquemas de las figuras 1 y 3 se pueden sacar conclusiones de tipo práctico y explicar empíricamente hechos que se dan en la técnica de la fabricación del clínker portland. Tales son:

a) la conveniencia de una gran finura y homogeneidad en los crudos;

b) la necesidad de que éstos estén sometidos a las adecuadas temperaturas de clinkerización durante los tiempos mínimos precisos; 
c) la de que se forme suficiente cantidad de fase líquida (función de los componentes $\mathrm{Al}_{2} \mathrm{O}_{3}, \mathrm{Fe}_{2} \mathrm{O}_{3}, \mathrm{MgO}$ y álcalis $-\mathrm{K}_{2} \mathrm{O}+\mathrm{Na}_{2} \mathrm{O}$ - principalmente), pero no excesiva, para que la formación del silicato tricálcico $\mathrm{C}_{3} \mathrm{~S}$ transcurra con velocidad apropiada;

d) la conveniencia de evitar la acción nociva y perturbadora de la incorporación de cenizas de carbón, sobre todo en la zona de clinkerización, pese a que la acción química de las mismas se tenga en cuenta a la hora de dosificar el crudo;

e) el buen resultado que, en general, da el enfriamiento rápido (templado) del clínker en diversos aspectos de la utilización del cemento;

f) como colofón de todo lo anterior, la conveniencia de granular el crudo lo más menudo posible, en compatibilidad con otras necesidades técnicas de la fabricación, a fin de hacer que la relación de zonas corticales a internas adquiera el valor máximo en todo el conjunto del crudo granulado.

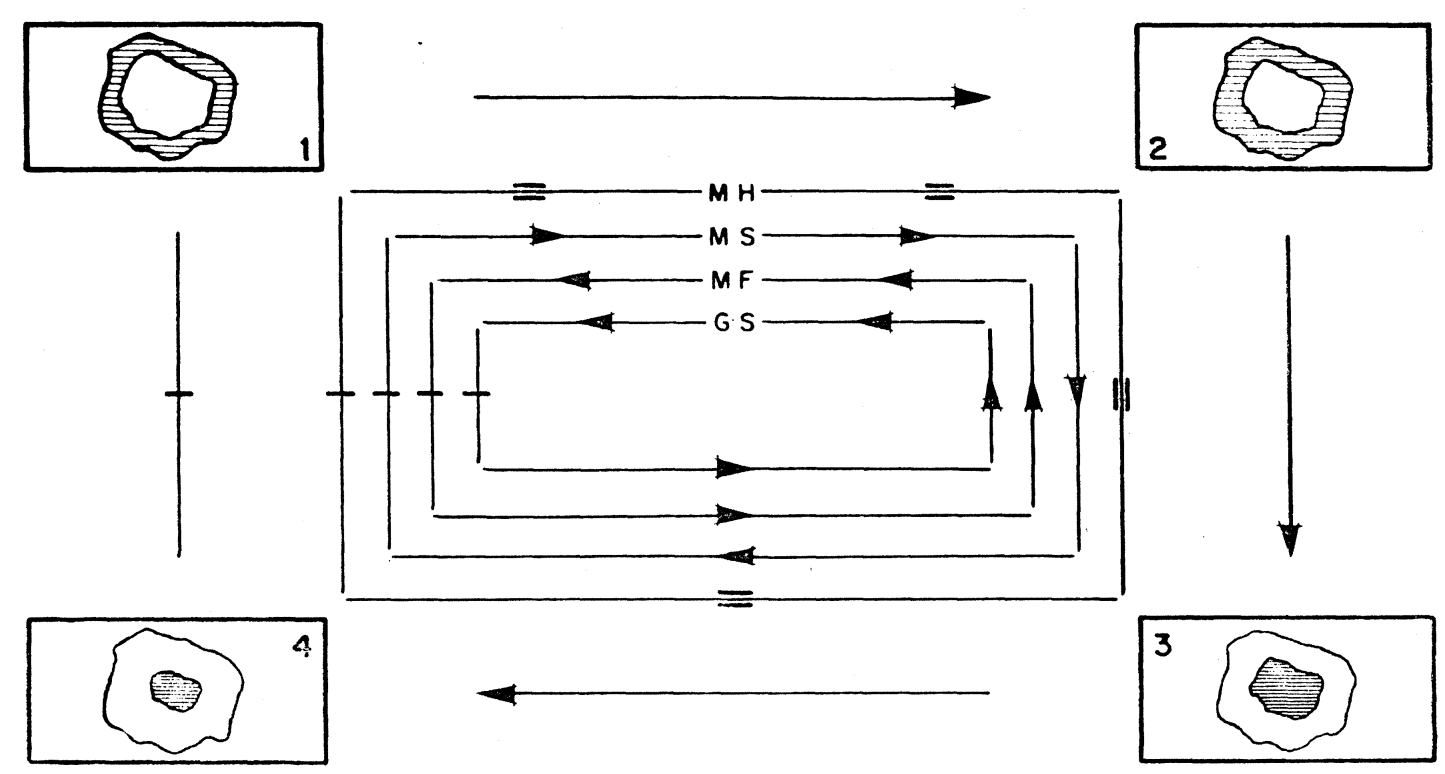

Fig. 3

Todos estos hechos y conclusiones, así como otros de no menor importancia, se ven notablemente reforzados por los resultados que pone de relieve el esquema de la figura 2 (incluidos también en la figura 4). Pero a estos resultados se ha llegado por vía de cálculo, aplicando las fórmulas de Dahl y Bogue (5) y presuponiendo unas ciertas hipótesis, y algunos de dichos resultados (sobre todo los relativos a las zonas internas de los gránulos), según el correspondiente estudio estadíśíico, presentan unas dispersiones elevadas, particularmente los que se refieren a la distribución de los silicatos $\mathrm{C}_{3} \mathrm{~S}$ y $\mathrm{C}_{2} \mathrm{~S}$ (3). Aparte de esto, las fórmulas de Dahl y Bogue sólo son aplicables en rigor cuando los constituyentes del clínker llegan a alcanzar el equilibrio a la temperatura ordinaria, lo que exige un enfriamiento lentísimo del material, que en la técnica de la fabricación del clínker nunca se da. Tampoco la aplicación de las correcciones a las fórmulas de Dahl y Bogue hechas por Lea y Parker (6), cuando se considera el equilibrio bloqueado a $1450^{\circ} \mathrm{C}$, o la cristalización independiente, es enteramente factible en el caso del clínker industrial. 
Por estos motivos, toda conclusión fundada en el esquema de la figura 2 debe ir refrendada por los correspondientes hechos experimentales, por lo que se impone, en primer lugar, confirmar si lo destacado en dicho esquema responde a una realidad en la práctica.

De igual manera, sólo una indudable y experimental puesta en evidencia de dichos hechos puede permitir una interpretación de los mismos más racional y en consonancia con los resultados de los estudios basados en los diagramés de fases. Para ello resultan insuficientes las técnicas químicas usuales y es preciso recurrir a otras de naturaleza más bien física. Tales son la nicroscopía petrográfica y la difracción de rayos $\mathrm{X}$, la primera de las cuales es objeto del presente trabajo, como ya en otros lugares se anunció (3) y (4).

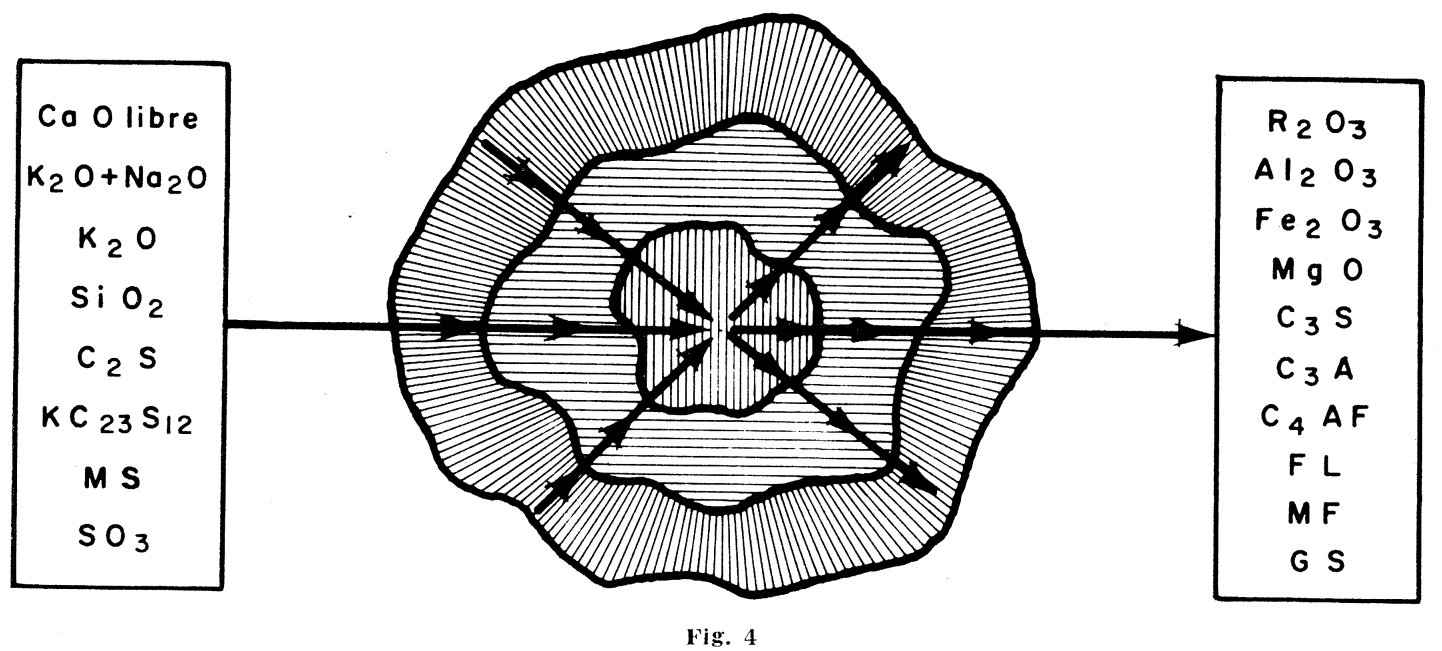

TECNICA EXPERIMENTAL

\section{II.1 Fundamento del método}

En un aspecto mineralógico cualitativo, el clínker granulado de cemento portland se asemeja a una roca artificial, cuya génesis es comparable hasta cierto punto con la de una roca natural, eruptiva o ignea. Es, por lo tanto, un conglomerado de fases cristalinas y fase vítrea, formado a partir de una masa reaccionante parcialmente fundida, en la cual las reacciones se verifican tanto entre las fases sólidas como entre éstas y el magma fundido existente en cada momento.

A la observación del clínker y a la identificación de las especies mineralógicas que como tal roca artificial la constituyen, son aplicables, por lo tanto, las técnicas de la microscopía petrográfica.

En el aspecto mineralógico cuantitativo pueden ser también de aplicación dichas técnicas, siempre y cuando se den las condiciones generales precisas para su aplicabilidad. Dichas condiciones son las siguientes:

a) que el material observado sea petrográficamente homogéneo;

b) que no contenga constituyentes mineralógicos desarrollados en un sentido determinado ni orientados según direcciones privilegiadas;

c) que no exista mucha diferencia entre los tamaños de los distintos cristales y, en todo caso, que éstos, en conjunto, no sean demasiado pequeños; 
d) que la extensión superficial de la zona observada guarde relación (proporcionalidad directa) con el diámetro medio del constituyente de mayor tamaño.

Sólo así podrá existir verdadera igualdad en todos los casos entre las siguientes relaciones:

1 - la relación de la longitud cubierta por cada especie mineral en una dirección determinada de la preparación microscópica, y la longitud total recorrida en el recuento (que es lo que realmente se mide);

2 - la relación de la superficie ocupada por dicha especie y la superficie total correspondiente en la sección plana o delgada observada (que es la que en verdad se prepara para el recuento);

3 - la relación del volumen ocupado por la especie en cuestión y el correspondiente volumen total (que es lo que en realidad se obtiene en el recuento microscópico).

La igualdad de estas tres relaciones define una proporcionalidad, y con ella hay posibilidad de obtener tantos por ciento de minerales en volumen, a partir de mediciones de longitud, fundamento en que se basan las platinas integradoras. De los tantos por ciento en volumen se pasa después a los ponderales si se conoce o si se determina el peso específico de cada constituyente.

En la práctica, el clínker rara vez cumple con las condiciones a) y c), pues es frecuente observar zonas en que aparecen agrupados cristales de belita, y otras, en cambio, preponderantemente alíticas; también sucede que los cristales de todos los constituyentes en general, y los de las fases intersticiales en particular, son muy pequeños. Lo primero ocurre porque la homogeneidad de los crudos de cemento, aun en el mejor de los casos, dista de ser perfecta.

Por todo ello existe una diferencia notable entre la observación microscópica de una roca natural y la del clínker: en aquélla es fácil determinar la composición cualitativa y cuantitativa, y difícil sacar conclusiones respecto de su génesis; en el clínker, de composición más constante, sucede lo contrario.

De todos modos, para evitar al máximo los inconvenientes que pueden emanar del incumplimiento de las condiciones a) y c), se aconseja, y así se ha hecho en el presente trabajo, hacer numerosos recuentos en diversas zonas de las preparaciones y sacar unos valores medios representativos. Por otra parte, el kocho de la heterogeneidad petrográfica del material es en el caso presente una ventaja, pues precisamente dicha heterogeneidad es la que se trata de poner de manifiesto para deducir de ella conclusiones de carácter tecnológico relacionadas con la calidad del clínker y con los procesos que intervienen en su fabricación.

\section{II.2 Preparación de los gránulos de clínker y observación microscópica de los mismos}

\section{II.2.1 Desbaste y pulido}

Se utilizaron gránulos de clínker de diversas procedencias, de tamaños comprendidos entre 6 y $10 \mathrm{~mm}$. Se desbastaron los gránulos mediante una rueda de esmeril (fig. 5) hasta conseguir una sección central en cada uno, la cual se pulió en etapas sucesivas, empleando para ello una máquina pulidora Buhler (fig. 6) y abrasivos de acción gradual, a saber: discos de papel de esmeril Buhler de tamaños de grano comprendidos entre 3 y 000 ; pa- 
ño de terciopelo impregnado en etanol absoluto para eliminar los restos del esmeril; paño de algodón impregnado en etanol absoluto. Al pasar de un grado de esmeril al siguiente se efectuaba el nuevo pulido en una dirección normal a la del anterior hasta eliminar totalmente las estrías producidas por éste.

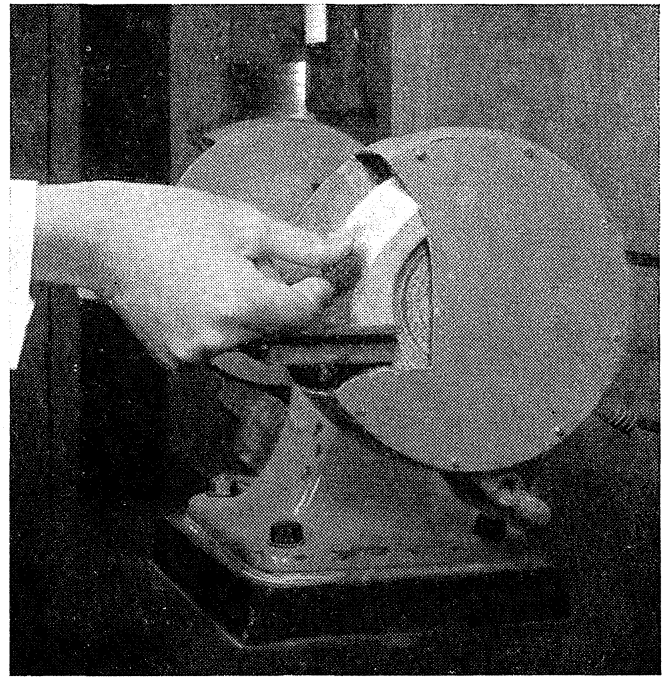

Fig. 5

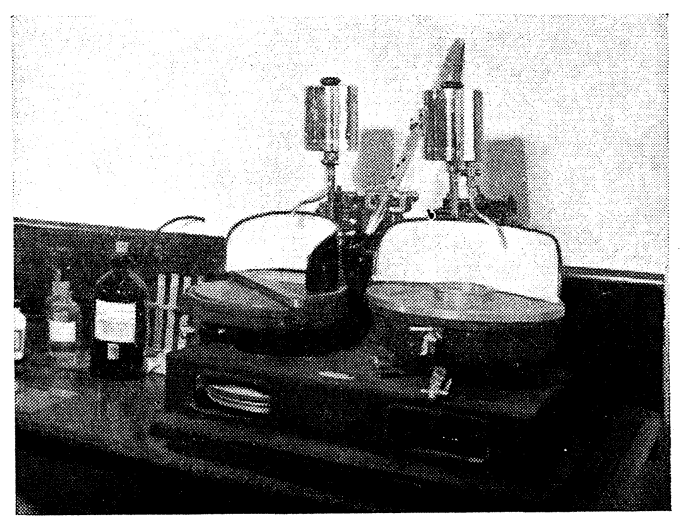

Fig. 6

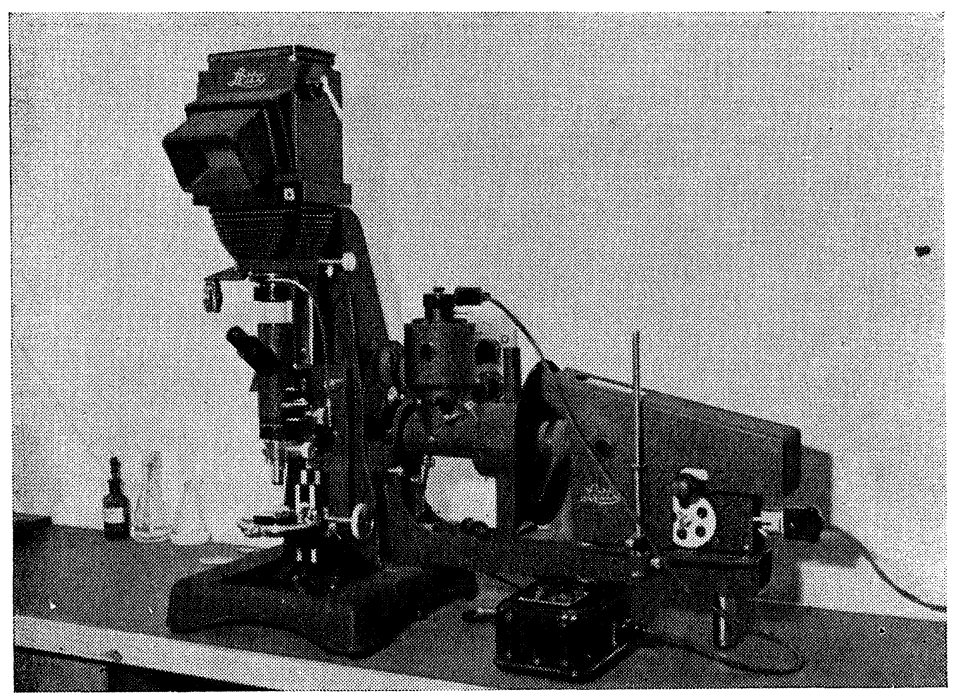

Fig. 7

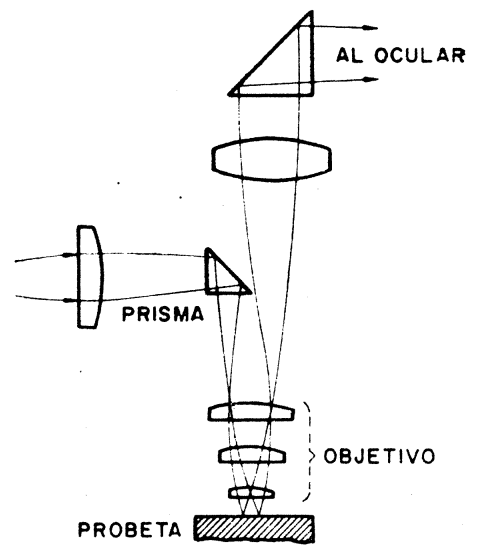

Fig. 8

\section{II.2.2 Observación microscópica}

La observación microscópica se hizo por reflexión utilizando para ello un microscopio universal Leitz, modelo "Panphot" (fig. 7). La óptica empleada fue: ocular $10 \times$ y objetivo $35 \times$ de apertura numérica 0,85 . La iluminación se consiguió mediante el "Opak" de polarización Leitz para luz incidente (fig. 8). Con la citada óptica se consigue un aumento de $400 \times$, aproximadamente. 


\section{II.2.3 Fotomicrografías}

El microscopio "Panphot" Leitz (fig. 7) permite observar la imagen del campo enfocado, proyectada sobre un vidrio deslustrado, y obtener fotografías de dicha imagen en placas. Esto se efectuó en aquellos casos en que interesaba destacar alguna particularidad del clínker observado, como se indica en el lugar correspondiente.

Se utilizaron placas Walka "autográficas" ortocromáticas. La iluminación fue proporcionada por la lámpara normal de tungsteno del equipo Leitz (corriente de $6 \mathrm{~V}$ y $5 \mathrm{~A}$ ) y el tiempo de exposición fue de 45 segundos. Como revelador se empleó el de alto contraste Kodak D-19.

El aumento conseguido en las fotomicrografías, dadas las características de la cámara usada es, aproximadamente, de $600 \times$.

\section{II.2.4 Recuentos planimétricos de los constituyentes mineralógicos del clínker}

La determinación cuantitativa de los constituyentes mineralógicos se llevó a cabo por el procedimiento clásico de la platina integradora, utilizándose a tal efecto una platina Leitz de seis tornillos micrométricos, con un grado de apreciación de 0,01 mm (figura 9).

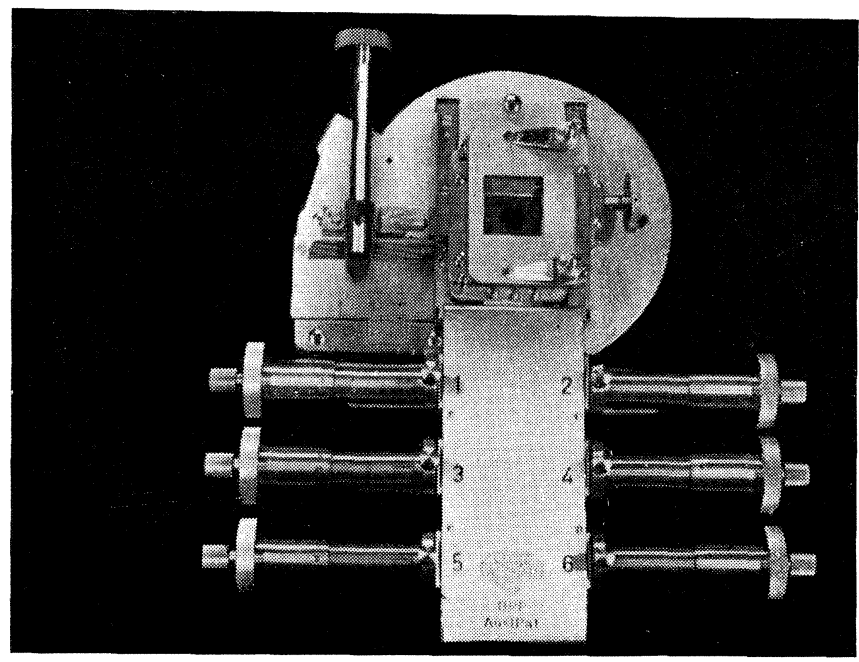

Fig. 9

Los constituyentes determinados cuantitativamente han sido: alita, que en el caso presente se homologa como silicato tricálcico $\mathrm{C}_{3} \mathrm{~S}$ y se expresa como tal; belita, homologada como silicato bicálcico $\mathrm{C}_{2} \mathrm{~S}$ y expresada como tal; celita oscura (rectangular - celita a - o prismática), homologada en conjunto como aluminato tricálcico $\mathrm{C}_{3} \mathrm{~A}$ y expresada como tal; celita clara (celita b), homologada como ferritoaluminato tetracálcico $\mathrm{C}_{4} \mathrm{AF}$ y expresada como tal; cal libre: este constituyente sólo se ha recontado cuando se trata de cal libre primaria, pues la secundaria aparece muy dispersa y la pequeñez de sus individuos invalidaría grandemente los resultados que se obtuvieran, dada la condición c) expuesta en II.1, la cual, evidentemente no se cumple. 
La magnesia libre no se ha determinado, en primer lugar por su escaso contenido total en los clínkereś estudiados y, en segundo, por no aparecer en forma de periclasa, sino en disolución sólida con otros constituyentes, lo que denota un enfriamiento rápido de dichos clínkeres e imposibilita el recuento del citado componente.

En cambio, se efectuó la determinación cuantitativa de los poros o huecos encontrados en los gránulos a lo largo de las líneas de medición, lo que ha permitido determinar la porosidad del material y la distribución de ésta en las distintas zonas de los gránulos. Para el recuento de los poros se asignó a éstos uno de los tornillos de la platina integradora, como si se tratara de un constituyente más, con lo cual los seis tornillos de la misma tuvieron su asignación correspondiente.

La forma de efectuar las mediciones de los seis constituyentes considerados se explica a la vista del esquema gráfico de la figura 10. En ella se representa la sección plana, pulida y atacada, de uno de los gránulos de clínker, lista para la observación y el recuento. Esta sección que es ampliación de la real del gránulo a escala aproximada de $20: 1(20 \times)$, se construyó determinando la posición de los puntos $A_{1}, B_{1} \ldots ; A^{\prime}{ }_{1}, B^{\prime}{ }_{1} \ldots$, a partir de un origen fijo $P$ de coordenadas conocidas y determinadas mediante las escalas rectangulares de la platina. Estas escalas, por medio de juegos de tornillo y cremallera, se desplazan con movimientos independientes de los originados por los tornillos micrométricos. Se escoge en el campo visual el punto $P$ correspondiente a la parte más "oriental" del gránulo y se fija de antemano la posición del porta que sostiene a éste fijo y horizontal mediante un soporte de plastilina, de forma que la coordenada horizontal $h$ de dicho punto coincida con el origen de la correspondiente escala y sea posible, por lo tanto, el máximo desplazamiento en dirección "este-oeste" según la línea $P Q$. La coordenada vertical $v$ de dicho punto se fija, también de antemano, aproximadamente hacia la mitad del margen de recorrido en la dirección "norte-sur", de manera que sea posible determinar distancias hacia el "norte" $\mathrm{N}$ y hacia el "sur" $\mathrm{S}$, a partir de la línea de cero o "ecuatorial" $P Q$.

Determinadas y anotadas las coordenadas del punto de origen $P$ de forma que pueda ser identificado y situado en cualquier momento en tales coordenadas, para lo cual es imprescindible que, una vez hecho el ajuste, la preparación no sufra ningún desplazamiento sobre la parte de la platina en que se apoya, se desplaza la preparación hacia la derecha (en dirección "este") a intervalos regulares e iguales a $0,5 \mathrm{~mm}$ (a $1 \mathrm{~cm}$ en la ampliación a $20 \times$, según la figura 10), operando para ello sobre el juego de tornillo y cremallera correspondiente a la escala horizontal $h$.

En cada uno de estos intervalos que definen los puntos $a, b, \ldots$ se miden las distancias de los puntos $A_{1}, A^{\prime}{ }_{1} ; B_{1}, B^{\prime}{ }_{1} \ldots$ situados respectivamente sobre los bordes superior ("norte") e inferior ("sur") del gránulo, a la línea de cero $P Q$ que contiene la sucesión de puntos $a, b \ldots$

De este modo se pueden situar, a la escala que convenga (20:1 en la figura 10) y preferentemente sobre papel milimetrado, las posiciones de los puntos $A_{1}, B_{1} \ldots ; A^{\prime}{ }_{1}, B^{\prime}{ }_{1} \ldots$, con lo cual se tiene determinado y puede dibujarse a dicha escala el contorno o borde del gránulo observado.

Para establecer en toda la superficie del gránulo tres zonas (periférica o cortical 1, intermedia 2, e interna o nuclear 3) como se indica en I, se procede del modo siguiente: Sobre el esquema ampliado, representativo del gránulo según la figura 10 , se fija aproximadamente un punto $Z$ que pueda considerarse como centro geométrico de la figura. A partir de él, y en diversas direcciones radiales (que, siguiendo el simil de la "rosa de los vientos" podrían ser ocho: $\mathrm{N} ; \mathrm{S} ; \mathrm{E} ; \mathrm{O} ; \mathrm{NE} ; \mathrm{SE}$; SO; NO; tal como se indica en lá 


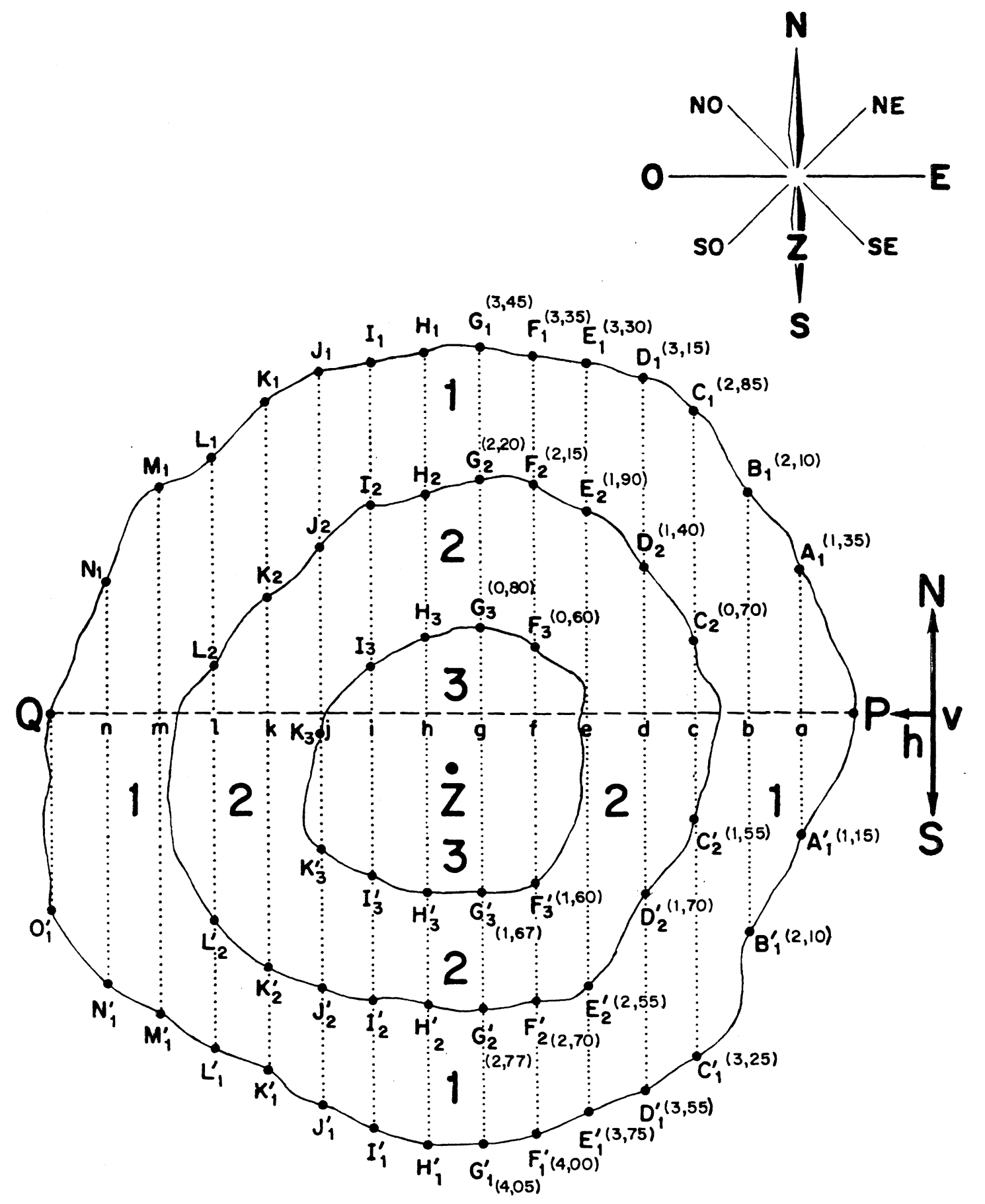

ESCALA 20:1

Fig. 10 
figura 10) se miden las distancias a los correspondientes puntos de la periferia del gránulo. Estas distancias se dividen por 3 y a partir del punto $Z$, y en las mismas direcciones antes indicadas, se fijan puntos situados a $1 / 3$ y $2 / 3$ de dichas distancias a partir de $\mathrm{Z}$. El conjunto de cada grupo de puntos así situado nos determina en el esquema dos contornos interiores homotéticos del perímetro del gránulo respecto del punto $Z$ (centro de homotecia).

El paso siguiente consiste en situar sobre el contorno intermedio los puntos $C_{2}, D_{2} \ldots$; $C^{\prime}{ }_{2}, D^{\prime}{ }_{2} \ldots$, y sobre el contorno interior los puntos $F_{3}, G_{3} \ldots ; F^{\prime}{ }_{3}, G^{\prime}{ }_{3} \ldots$ determinados por las coordenadas horizontales $c, d \ldots ; f, g \ldots$, a partir de $\mathrm{P}$, respectivamente. Con ello se pueden medir sobre el gráfico las distancias $c C_{2}, d D_{2} \ldots ; c C^{\prime}{ }_{2}, d D^{\prime}{ }_{2} \ldots$, cuyos valores, expresados en unidades de la escala, se anotan al lado de cada punto, tal como indica la figura 10 , en la que por sencillez se han anotado solamente los valores correspondientes a los puntos situados en la mitad derecha del esquema. Con éstos se puede proceder ya al recuento de constituyentes por zonas, de la forma que se expone a continuación.

Supuesta la preparación con el punto $P$ origen situado en el retículo del ocular, y a los seis tornillos micrométricos puestos en cero, se desplaza la platina mediante los sistemas de tornillo y cremallera de las escalas horizontal y vertical, primero desde $P$ hasta $a$ según la escala horizontal, y después desde $a$ hasta $A^{\prime}{ }_{1}$ según la vertical.

Situado el retículo en $A^{\prime}{ }_{1}$ y comprobado que los seis tornillos micrométricos permanecen en cero, se inicia el recorrido $A^{\prime}{ }_{1} \rightarrow A_{1}$, haciendo girar siempre en dicha dirección y sentido cada tornillo, cada vez que se sobrepase el constituyente que previamente le ha sido asignado. Terminado el recorrido en $A_{1}$, se anota el recorrido total efectuado con cada uno de los tornillos. La suma de estos recorridos debe ser igual a la distancia total entre $A^{\prime}{ }_{1}$ y $A_{1}$, es decir, a la suma de los valores $a A_{1}$ y $a A^{\prime}{ }_{1}$, previamente medidos y anotados al lado de cada uno de los respectivos puntos $A^{\prime}{ }_{1}$ y $A_{1}$. En caso de no coincidir la suma y la distancia total (dentro de un margen de tolerancia de $0,2 \mathrm{~mm}$ por ejemplo), deberá repetirse el recuento y, si aun así persiste la discrepancia, deberá verificarse el esquema.

Después de este primer recuento se dejan libres los tornillos micrométricos soltando para ello los de fijación, con lo cual la platina vuelve por sí sola a la posición de origen, debiendo situarse de nuevo el punto $A^{\prime}{ }_{1}$ en el retículo.

A continuación se procede al segundo recuento, con las mismas características del primero, entre $B^{\prime}{ }_{1}$ y $B_{1}$. En estos dos recuentos solamente se ha recorrido la zona 1 que se ha designado como externa o cortical.

El tercer recuento entre $C^{\prime}{ }_{1}$ y $C_{1}$ afecta ya a dos zonas: la 1 externa o cortical (entre $C^{\prime}{ }_{1}$ y $C^{\prime}{ }_{2}$, y $C_{2}$ y $C_{1}$ ) y la 2 intermedia (entre $C^{\prime}{ }_{2}$ y $C_{2}$ ). El recuento, por lo tanto, se divide en este caso en tres partes: de $C^{\prime}{ }_{1}$ a $C^{\prime}{ }_{2}$, de $C^{\prime}{ }_{2}$ a $C_{2}$ y de $C_{2}$ a $C_{1}$. La primera parte del recuento entre $C^{\prime}{ }_{1} \mathrm{y} \mathrm{C}_{2}{ }_{2}$ no ofrece, respecto de lo ya explicado, más particularidad que la de que éste debe concluir cuando el recorrido suma de los efectuados con todos los tornillos sea igual a la distancia $C^{\prime}{ }_{1} C^{\prime}{ }_{2}$ dada por la diferencia entre los valores numéricos situados a la derecha de dichos puntos. Esto exige la precaución de ir sumando los recorridos para no rebasar el valor de dicha diferencia.

Terminada la primera parte del recuento en $C^{\prime}{ }_{2}$ se anota el recorrido total efectuado con cada uno de los tornillos.

La segunda parte del recuento entre $C^{\prime}{ }_{2}$ y $C_{2}$ podría hacerse a continuación, sin más que considerar como cero en cada tornillo el valor final resultante de la primera parte del re- 
cuento. De igual modo, la tercera parte del mismo podría hacerse a continuación de la segunda, de forma análoga.

Esto, que sería lo inmediato, tiene como limitación la llegada a tope de uno cualquiera de los tornillos antes de haber alcanzado, bien sea el punto $C_{2}$, o bien el $C_{1}$, lo cual invalidaría el recuento en la correspondiente etapa. Por ello es preferible operar de la manera que se indica en lo que sigue.

Concluida la primera parte del recuento en $C^{\prime}{ }_{2}$ y anotados los recorridos totales efectuados con los tornillos, se dejan libres éstos, con lo cual la platina vuelve por sí misma a la posición de origen, situándose el punto $C^{\prime}{ }_{1}$ en el retículo del ocular. Puestos los dos tornillos micrométricos en cero, se desplaza la platina desde $C^{\prime}{ }_{1}$ hasta $C^{\prime}{ }_{2}$, por medio del tornillo y la cremallera correspondientes a la escala vertical de la misma.

A partir de este punto y hasta $C_{2}$ se procede a la segunda parte del recuento, en la forma que ya queda expuesta. Alcanzado $C_{2}$, se anota nuevamente el recorrido total realizado con cada uno de los tornillos, se dejan éstos libres, con lo que la platina vuelve por sí sola a la posición de origen (en este caso $C_{2}^{\prime}$ ), situándose este punto en el retículo. Puestos los tornillos en cero, se desplaza la platina desde $C^{\prime}{ }_{2}$ hasta $C_{2}$, mediante el tornillo y la cremallera correspondientes a su escala vertical.

A partir de $C_{2}$ y hasta $C_{1}$ se efectúa del modo indicado en la tercera parte del recuento.

De la misma manera que entre $C^{\prime}{ }_{1}$ y $C_{1}$, se procede entre $D_{1}^{\prime}$ y $D_{1}, E^{\prime}{ }_{1}$ y $E_{1}$, etc. Las líneas desde $F^{\prime}{ }_{1} F_{1}$ hasta $J^{\prime}{ }_{1} J_{1}$ atraviesan las tres zonas 1 , 2 y 3 en cinco etapas sucesivas: primera, de $F_{1}^{\prime}{ }_{1}$ a $F_{2}^{\prime}$; segunda, de $F_{2}^{\prime}{ }_{2}$ a $F_{3}^{\prime}$; tercera, de $F_{3}^{\prime}$ a $F_{3}$; cuarta, de $F_{3}$ a $F_{2}$, y quinta, de $F_{2}$ a $F_{1}$, por ejemplo. En cada una de estas etapas se procede de forma análoga a como ya queda descrito para la línea $C^{\prime}{ }_{1} C_{1}$.

Acabados los recuentos a lo largo de todas las líneas, se agrupan los correspondientes a cada una de las tres zonas distintas, sumando los recuentos parciales en la forma que se indica en el cuadro 1.

CUADRO 1

\begin{tabular}{|c|c|c|}
\hline Zona 1 & Zona 2 & Zona 3 \\
\hline 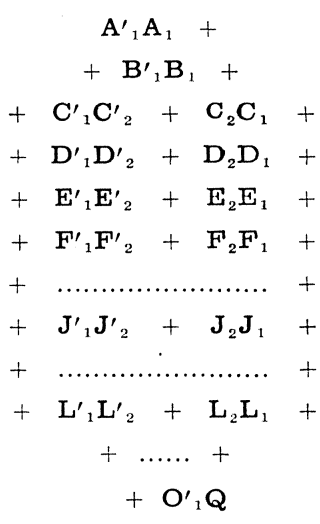 & $\begin{array}{c}\mathrm{C}_{2}{ }_{2} \mathrm{C}_{2}+ \\
+\mathrm{D}_{2}{ }_{2} \mathrm{D}_{2}+ \\
+\mathrm{E}_{2}{ }_{2} \mathrm{E}_{2}+ \\
+\mathrm{F}_{2}^{\prime} \mathrm{F}^{\prime}{ }_{3}+\mathrm{F}_{3} \mathrm{~F}_{2}+ \\
+\ldots \ldots \ldots \ldots \ldots \ldots \ldots \ldots+ \\
+\mathrm{J}_{2} \mathrm{~K}^{\prime}{ }_{3}+\mathrm{K}_{3} \mathrm{~J}_{2}+ \\
+\ldots \ldots \ldots \ldots \ldots \cdots \cdots+ \\
+\mathrm{L}_{2} \mathrm{~L}_{2}\end{array}$ & $\begin{array}{l}\mathrm{F}_{3}^{\prime}{ }_{3} \mathrm{~F}_{3}+ \\
+\ldots \ldots+ \\
+\mathrm{K}_{3}{ }_{3} \mathrm{~K}_{3}\end{array}$ \\
\hline
\end{tabular}




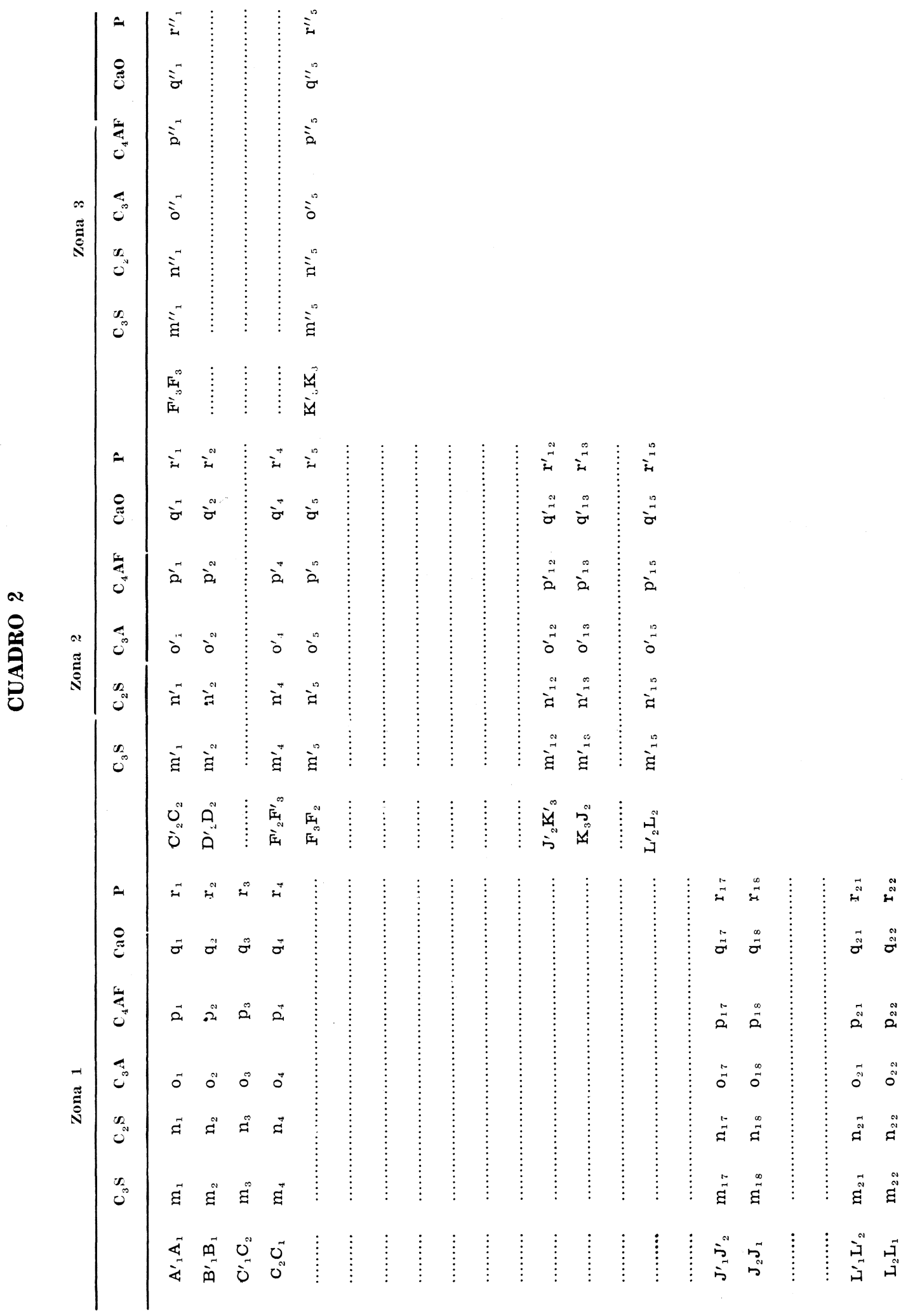


Naturalmente, cada uno de los sumandos anteriores se compone a su vez de seis, que son los seis constituyentes recontados, con lo cual las sumas esquematizadas antes adoptarían en realidad la forma esquematizada en el cuadro 2, siendo $m, n, o, p, q, r$, en cada caso, los recorridos parciales correspondientes a cada uno de los constituyentes $\mathrm{C}_{3} \mathrm{~S}, \mathrm{C}_{2} \mathrm{~S}, \mathrm{C}_{3} \mathrm{~A}$, $\mathrm{C}_{4} \mathrm{AF}$, cal libre $(\mathrm{CaO})$ y poros $(\mathrm{P})$, respectivamente.

El tanto por ciento de espacio longitudinal (y según lo expuesto en II.1 de espacio en superficie, e incluso en volumen) ocupado por cada constituyente, vendrá dado: para la zona 1 por el cociente $m_{1}+m_{2}+\ldots+m_{22} / A^{\prime}{ }_{1} A_{1}+B^{\prime} B_{1}+\ldots+L_{2} L_{1}$ multiplicado por 100, para el $\mathrm{C}_{3} \mathrm{~S}$; por el cociente $n_{1}+n_{2}+\ldots+n_{22} / A^{\prime}{ }_{1} A_{1}+B^{\prime}{ }_{1} B_{1}+\ldots+L_{2} L_{1}$ multiplicado por 100, para el $\mathrm{C}_{2} \mathrm{~S}$, etc.; para la zona 2, por el cociente $m_{1}^{\prime}{ }_{1}+m^{\prime}{ }_{2}+\ldots+$ $+m^{\prime}{ }_{15} / C_{2}^{\prime} C_{2}+D_{2}^{\prime} D_{2}+\ldots+L_{2}^{\prime} L_{2}$ multiplicado por 100 , para el $\mathrm{C}_{3} \mathrm{~S}$; por el cociente $n^{\prime}{ }_{1}+n^{\prime}{ }_{2}+\ldots+n^{\prime}{ }_{1:} / C_{2}^{\prime} C_{2}+D_{2}^{\prime} D_{2}+\ldots+L_{2}^{\prime} L_{2}$ multiplicado por 100 , para el $C_{2} \mathrm{~S}$, etcétera; para la zona 3 , por el cociente $m^{\prime \prime}{ }_{1}+\ldots+m^{\prime \prime}{ }_{z} / F^{\prime}{ }_{3} F_{3}+\ldots+K_{3}{ }_{3} K_{3}$ multiplicado por 100 , para el $\mathrm{C}_{3} \mathrm{~S}$; por el cociente $n^{\prime \prime}{ }_{1}+\ldots+n^{\prime \prime}{ }_{5} / F_{3}^{\prime} F_{3}+\ldots+K_{3}{ }_{3} K_{3}$ multiplicado por 100 , para el $\mathrm{C}_{2} \mathrm{~S}$, etc.

Se tienen así los tantos por ciento en volumen de cada uno de los seis constituyentes, en cada una de las tres zonas. Uno de estos constituyentes corresponde a poros o huecos, con lo cual se tiene directamente la porosidad en tanto por ciento, relativa a cada zona.

Si se desea tomar en consideración dos zonas más del gránulo, una constituida por el promedio de la zona externa y la media (a la que puede llamarse zona externa ampliada) y otra constituida por el promedio de la zona media y la interna (a la que puede llamarse zona interna ampliada), es preciso calcular los correspondientes promedios.

Para ello no es correcto hallar las medias aritméticas de las composiciones en las dos zonas promediadas sino que es preciso calcular unas medias ponderales (referidas todavía a composiciones en volumen), multiplicando cada composición por la superficie total de la zona a que corresponde (medida sobre el gráfico a escala), sumando los productos y dividiendo la suma resultante por la superficie suma de las correspondientes a las zonas consideradas. La forma de promediar zonas es la indicada en el cuadro 3, para dos zonas, 1 y 2, de superficies totales $S_{1}$ y $S_{2}$.

\section{CUADRO 3}

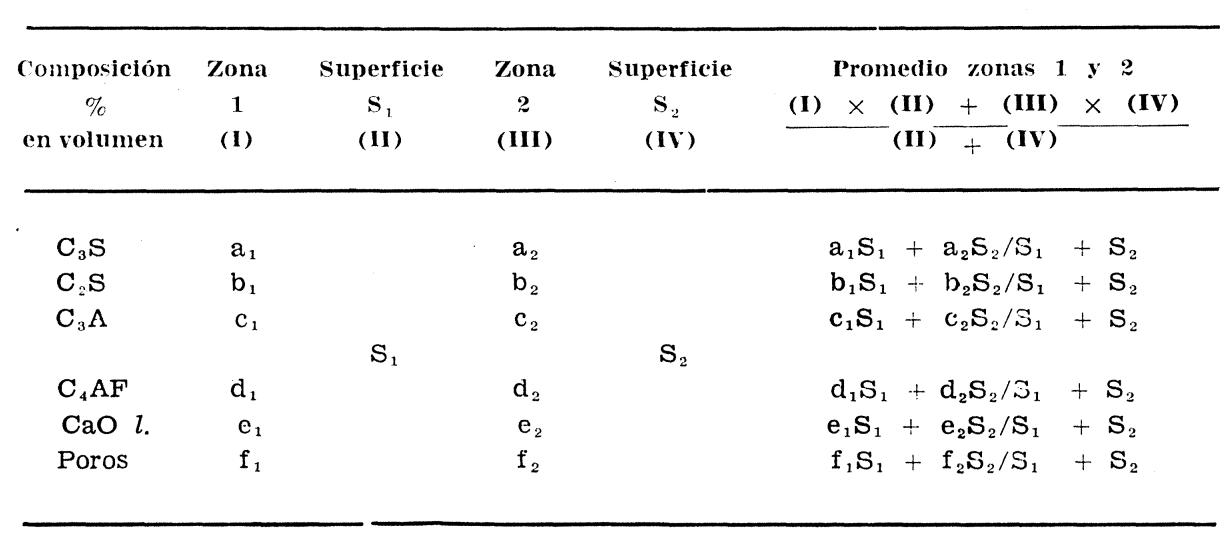

En lugar de operar con las superficies totales $S_{1}, S_{2}$ y $S_{3}$ de cada zona, lo cual requeriría la medición de éstas en el gráfico, planimétricamente o por pesada, pueden utilizarse las longitudes totales resultantes de los recuentos lineales efectuados en cada una de ellas, longitudes que ya se tienen o son fáciles de calcular (cuadro 1). La disposición de cálculo 
sería la misma indicada en el cuadro 3 , sin más que sustituir las superficies $S_{1}$ y $S_{2}$ por las longitudes totales recorridas en cada zona, $L_{1}$ y $L_{2}$.

Como se verá al exponer los resultados, los que se obtienen operando de una u otra forma son sorprendentemente concordantes, lo que denota la idoneidad del método al cumplirse las condiciones básicas señaladas en II.1, dada la gran extensión de las mediciones efectuadas, lo cual es una garantía del valor representativo de dichos resultados.

Para obtener el tanto por ciento en peso de cada uno de los constituyentes en las distintas zonas, a excepción de los poros, se multiplican sus tantos por ciento en volumen por sus respectivas densidades, dadas en el cuadro 4, sacadas de la bibliografía (7).

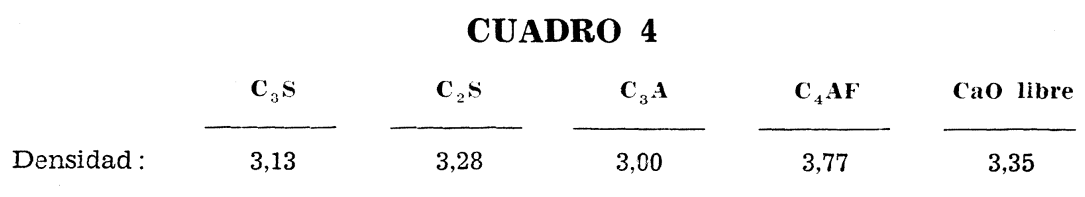

Se suman los producios así obtenidos y cada uno de ellos se expresa como tanto por ciento de la suma total. Con esto queda efectuado el recuento planimétrico de los constituyentes del gránulo por zonas, y expresados los resultados en tanto por ciento en peso de dichos constituyentes, referidos a material sólido (descontados los huecos o poros).

\section{bibliografia}

(1) Calleja, J.: Contribución al estudio del clinker de cemento Portland: Heterogeneidad en la distribución de la cal libre. An. Real Soc. Esp. Fís. y Quím. B XLVIII, 351 (1952). Materiales de Construcción (I.E.T.C.C.), núm. 24 (1952).

(2) Calleja, J.: Los álcalis en la estructura del clinker de cemento Portland. Revista de Ciencia Aplicada XIV, 397 (1959). Materiales de Construcción (I.E.T.C.C.), núms. 96 y 97 (1959-1960).

(3) Calleja, J.: Contribución al estudio de la estructura del clínker de cemento Portland. Monografía número 146 del I.E.T.C.C. (1954). Revista de Ciencia Aplicada IX, $202 ; 422 ; 524$ (1955). Materiales de Construcción (I.E.T.C.C.), núms. 55 a 64 (1954 - 1955).

(4) Calleja, J.: Sobre la estructura del clinker de cemento Portland. Memoria III Reunión Internacional Reactividad de Sólidos, Madrid, 1956. Materiales de Construcción (I.E.T.C.C.), núm. 80.

(5) Bogue, R.: The Chemistry of Portland Cement (1957). 2.a ed. New York (1955).

(6) Lea, F. M. y PARker, T. W. en Bogue (cita 5) y en LeA, F. M. y Desch, C. H.: The Chemistry of Cement and Concrete. 2. ${ }^{\mathrm{a}}$ ed. London (1956).

(7) Fuster, J.: Curso de Especialización en Cemento. (I.E.T.C.C.): Técnicas y Procedimientos Petrográficos, Madrid (1950). 